\title{
Structural and Optical Properties of AlGaN MQWs Grown by MOCVD Using One and Two TMG Sources
}

Shuo Wang ${ }^{1}$, Hongen Xie ${ }^{1}$, Yong O. Wei ${ }^{1}$, Alec M. Fischer ${ }^{1}$, Fernando A. Ponce ${ }^{1}$, M. Moseley ${ }^{2}$, B. Gunning $^{2}$, and W. A. Doolittle ${ }^{2}$

1. Department of Physics, Arizona State University, Tempe AZ 85287-1504, U.S.A

2. Advanced Semiconductor Technology Facility, School of Electrical and Computer Engineering, Georgia Institute of Technology, Atlanta, Georgia 30332, U.S.A.

Quantum wells are used to confine the electron-hole pairs and thus increase the quantum efficiency. However, the growth of a good-quality quantum well (QW) is challenging. Many factors such as choice of substrate, growth temperature, and number of sources affect the structure of the QWs. In this study, QWs are grown by metal-organic chemical vapor deposition (MOCVD) at a temperature of $1155{ }^{\circ} \mathrm{C}$, with one and two trimethylgallium (TMG) sources. The differences between one and two TMG sources are schematically shown in Figure 1. In one TMG configuration, the growth conditions between a quantum well and a quantum barrier (QB) requires the TMG flow rate to be interrupted and changed. During this change, there is no Ga flowing onto the sample's surface. In two TMG configuration, QW and QB are grown by two TMG sources with different flow rate. The active region growth switches between QW and QB without interruption. The aluminum content in the QWs and the QBs in both samples (using one and two TMG) are targeted to be 0.6 and 0.75 , respectively. Their structures are studied by scanning transmission electron microscope (STEM) and the optical properties by cathodoluminescence (CL).

Figure 2(a) shows a high-angle annular dark-field (HAADF) image of the QWs grown with one TMG source. The contrast in HAADF is proportional to $Z^{n}$, where $Z$ is the atomic number and $n=1.7-2$ [1]. There are dark layers at the interfaces between the QWs and the QBs. QWs have similar brightness as QBs. We can see three atomic layers in each dark layer from Fig. 2(b). Figure 2(c) shows a HAADF image of QWs grown with two TMG sources. There is no dark layers at the interfaces and the contrast difference between QWs and QBs is sharp, as shown in Fig. 2(d). Figure 3 shows the CL spectra of the two samples under the same excitation conditions. The sample grown by two TMG sources exhibits a CL emission intensity 7 times higher than the one grown by one TMG source. The emission peak position also changes from $263 \mathrm{~nm}(4.71 \mathrm{eV})$ to $257 \mathrm{~nm}(4.82 \mathrm{eV})$.

In the sample grown by one TMG source, the Al atoms are mostly gathered at the interfaces (dark layer in Fig. 2), which leads to a reduced quantum confinement as the width of the barriers is actually smaller than expected. This explains the lower efficiency of one TMG sample. The QWs grown with one TMG have a lower Ga content than $40 \%$ (less contrast in Fig. 2) which together with the Al-rich dark layers shift the ground state in the QW, leading to the observed emission peak blue shift [2].

References:

[1] P. D. Nellist and S. J. Pennycook, Adv. Imag. Electr. Phys. 113 (2000), p. 147.

[2] The authors gratefully acknowledge the use of facilities within the LeRoy Eyring Center for Solid State Science at Arizona State University. 

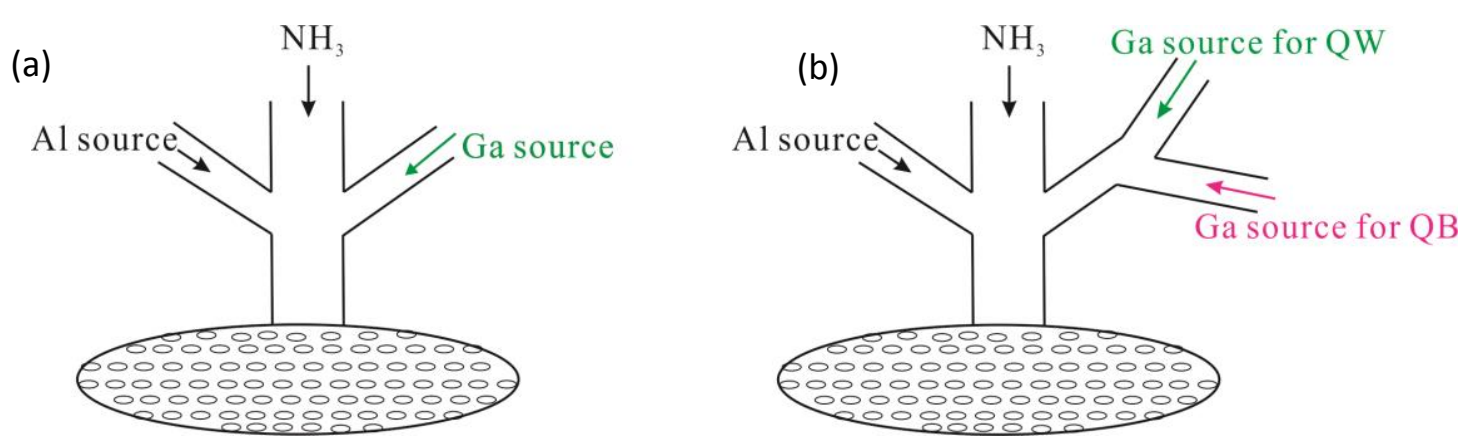

Figure 1. Schematic diagram of (a) one TMG source and (b) two TMG sources configuration.

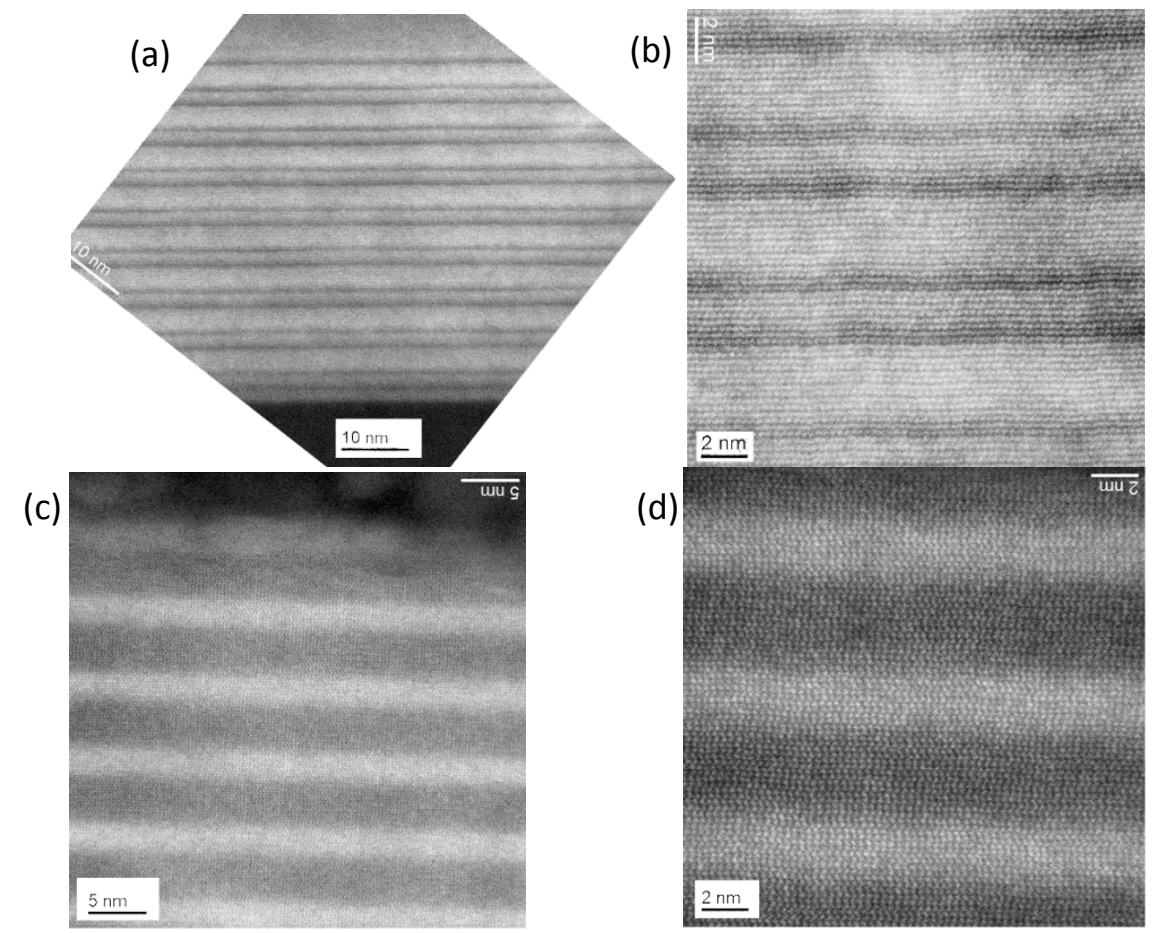

Figure 2. HAADF images of the QW region with (a) one TMG source and (c) two TMG sources. Higher magnification images are shown in (b) and (d), respectively.

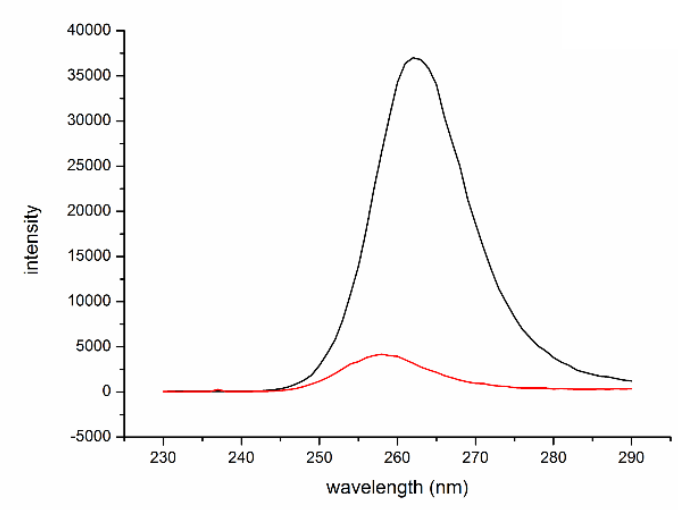

Figure 3. CL spectra of the QW emission by one TMG source (lower curve) and two TMG sources (upper curve). 